\title{
The interaction of arousal and retention interval: Ipsative vs normative scoring ${ }^{1}$
}

\author{
STEPHEN KAPLAN and RACHEL \\ KAPLAN, University of Michigan, Ann \\ Arbor, Mich. 48104
}

While the within-S, or ipsative, galvanic skin response (GSR) categorization has led to an arousal/retention-interval interaction with superior immediate recall of low-arousal items and reminiscence of high-arousal items, a different pattern of results has been reported using a normative GSR categorization. This mode of analysis, based on mean GSRs across all Ss, has vielded superior recall for high items across all time conditions. A reanalvsis of three paired-associate studies, previously published using the ipsative procedure. shows the normative pattern for the no studies involving meaningful material, but not in the case of nonsense-syllable stimulus items. It is concluded that both normative and ipsative effects can account for a significant portion of the variance in a (me-trial paired-associate paradigm using meaningtiul material. In comrast to the ipsative GSR, the nommatine GSR appears to be related to the way meaningfial items are characteristically enconded.

Several recent studies have demonstrated differential-recall levels as a function of galvanic skin response (GSR). Studies using the paired-associate paradigm, with stimulated recall procedure and relatively short lists, have shown that GSR and retention interval interact in their effect on recall (Kleinsmith \& Kaplan, 1963, 1964; Kaplan \& Kaplan, 1969). If memory is tested very soon after training, the low-arousal items are more frequently recalled; but if the memory test occurs $45 \mathrm{~min}$, a day, or even a week after training, it is the high-arousal items that are better recalled. These studies have based the GSR categorization on a within-S or ipsative comparison. Each S's GSRs are ranked, and his correct recall of the response elements is compared for those items that had originally been accompanied by high GSRs and for those that had shown low GSRs.

By contrast, several other studies have shown superior recall of high-arousal items, even if tested immediately after training (Maltzman, Kantor, \& Langdon, 1966; Yarmey, 1966: Kaplan, Kaplan, \& Sampson, 1968). The one methodological feature that the studies showing superior recall of high-arousal items have in common is that the categorization of items into high and low arousal was not on an ipsative basis. Rather, a normative procedure was used. The designations were based on a priori grounds in the cases of the Maltzman et al and the Yarmey studies and on the mean GSRs for each item across all $S s$ in the case of the Kaplan et al study.

Since there tends to be considerable variability across Ss in their initial GSRs to a set of items, the ipsative and the normative procedures would, in general, lead to different categorizations when applied to the same data. Three of our paired-associate studies, previously analyzed only in terms of ipsative GSR, provide an opportunity to make this comparison. Included in these analyses are the Kleinsmith and Kaplan studies, using meaningful (1963) and nonsense material (1964), and the Kaplan \& Kaplan (1969) study, which used meaningful items.

\section{METHOD}

The three studies examined here share a common paradigm. A single learning trial was used, the response items were single-digit numbers, the interpolated slides always contained four colors to be named out loud, each slide was presented for $4 \mathrm{sec}$, and Ss were given no set to learn. To test for retention, the stimulus word was presented, and $S$ attempted to provide the previously paired response digit.

While the studies differed in a number of respects, the three variables of particular relevance here are the type of material, the list length, and the interstimulus interval. Meaningful words of known differential arousal effects were used in the 1963 and 1969 studies, while the 1964 study used zero association value nonsense syllables. The earliest study used eight items, and the other two studies each involved six-item lists. While two color slides were standard across all three studies, the 1963 and 1964 studies involved a stimulus slide followed by the paired-associate slide, while the 1969 study dropped the stimulus-alone slide and consequently involved a 12-sec rather than a $16-\mathrm{sec}$ interstimulus interval. The total presentation time, therefore, for the 1963 study was $128 \mathrm{sec}$; for the 1964 study, it was $96 \mathrm{sec}$; and for the 1969 study, it was $72 \mathrm{sec}$.

In each of the studies, skin resistance was recorded during learning using a constant current, wide-band recording system (Kaplan \& Hobart, 1964). This system utilizes a $10-m \mathrm{~V}$, high-impedance servorecorder and a control unit which provides a 10-microamphere measuring current. A modified version of the Lykken zinc-zinc sulphate fingertip electrode (Kaplan \& Fisher, 1964) was attached to the first the third fingers of S's nonwriting hand.

Scoring Procedure

For each study, the same GSR values that had been used in the previously presented results were used to determine the mean GSR value for each item across all $S s$ in that study. (For the two earlier studies that had $16-\mathrm{sec}$ interstimulus intervals, the maximal drop in S's skin resistance occurring within $12 \mathrm{sec}$ of onset of a given stimulus item was scored and converted to percentage of basal resistance level. For the 1969 study, an 8-sec scoring interval was used.) The normative means were ranked, and the items having mean GSRs above the median were designated "high normative items," and those falling below the median were designated "low normative items." For the meaningful material, the low items turned out to be EXAM, LOVE, MONEY, and DANCE for the 1963 study and EXAM, MONEY, and SWIM for the 1969 study. The high items in both studies included RAPE, KISS, and VOMIT; SWIM was the fourth highest normative item in the 1963 study. For the nonsense syllables, JEX, LAJ, and TOV were in the low category, while CEF, QAP, and DAX were in the high category.

\section{RESULTS AND DISCUSSION}

One result, already evident, is that the meaningful items did not divide precisely as expected in the 1963 study. One would have expected LOVE rather than SWIM to fall in the "high" category. In fact, on an a priori basis, SWIM might be expected to yield the lowest mean GSR of any of these items!

In the left column of Fig. 1 are the results of the normative GSR analyses for the three studies (1963 at top, 1969 in the middle, and 1964 at bottom). The ipsative GSR analyses are presented in the right column to permit direct comparison for each study. 2 The finding that the high normative items show superior recall at all time conditions, and notably at the immediate test, is evident for the meaningful material. This superiority is significant at $p<.001$ in the case of each of the two studies $(F=6.66, \mathrm{df}=1 / 41$ for the 1963 study; $F=18.37, \mathrm{df}=1 / 85$ for the 1969 study). In the case of the nonsense syllables, by contrast, the results using the normative GSRs are essentially identical to those using the ipsative GSR, namely, a significant interaction with superior immediate recall of low-arousal items and a reminiscence of the high-arousal items $(F=6.42, \quad \mathrm{df}=2 / 33$, $\mathrm{p}<.001)$. 
Fig. 1. Per cent recall of paired associates as a function of normative GSR (left column) and ipsative GSR (right column) for three studies: Top plots are based on Kleinsmith \& Kaplan, 1963; middle plots are based on Kaplan \& Kaplan, 1969; bottom plots are based on Kleinsmith \& Kaplan, 1964. Meaningful stimulus items were employed in the studies plotted at top and in the middle, while nonsense material was employed for the study plotted at bottom. The high and low designations are based on median split in each case.

The findings presented in Fig. 1, as well as the analysis of several unpublished studies, suggest that the normative procedure predicts superior recall across all time conditions given meaningful material. On both theoretical and empirical grounds, however, meaningfulness of the material appears to be merely a necessary and not a sufficient condition for obtaining the normative effect. Levonian's reanalysis of his study using obviously meaningful traffic safety film (1968) indicates that there is no "normative effect" in his data; the normative curves are essentially identical to the ipsative ones he published. Unlike our studies where the material is presented with one verbal item at a time, the Levonian films present complex and multidimensional stimuli very likely to generate idiosyncratic and emotionally loaded trains of thought. For such material, as for some nonmeaningful material, one might expect more variability in GSR reaction.

In our previous studies, we have conceptualized the ipsative GSR as an indication of an arousal reaction that would tend to enhance perseverative consolidation of traces representing recently presented material. (The initially poor recall of the high-arousal material can be attributed to a temporary central fatigue generated by the same heightened perseveration that enhances consolidation. A fuller discussion of the fatigue concept can be found in Pomerantz, Kaplan, \& Kaplan, 1969.)

The normative results, however, require a quite different interpretation. It appears that meaningful material is encoded in certain characteristic ways, at least in the population studied, that is reflected in the normative scores. This encoding, in turn, appears to have an important influence on recall across a wide range of intervals. The emphasis on encoding here is based on the fact that this factor influences $S$ 's recall independent of his particular GSR to the item when it was presented. The failure of nonmeaningful material to show this relationship provides additional support for
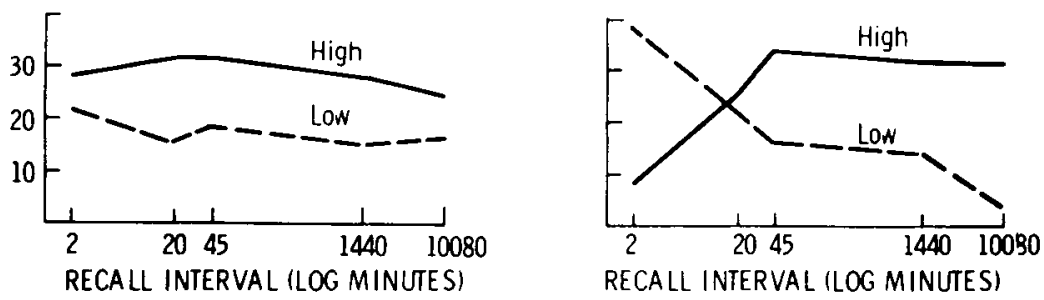

RECALL INIERVAL (LOG MINUTES)
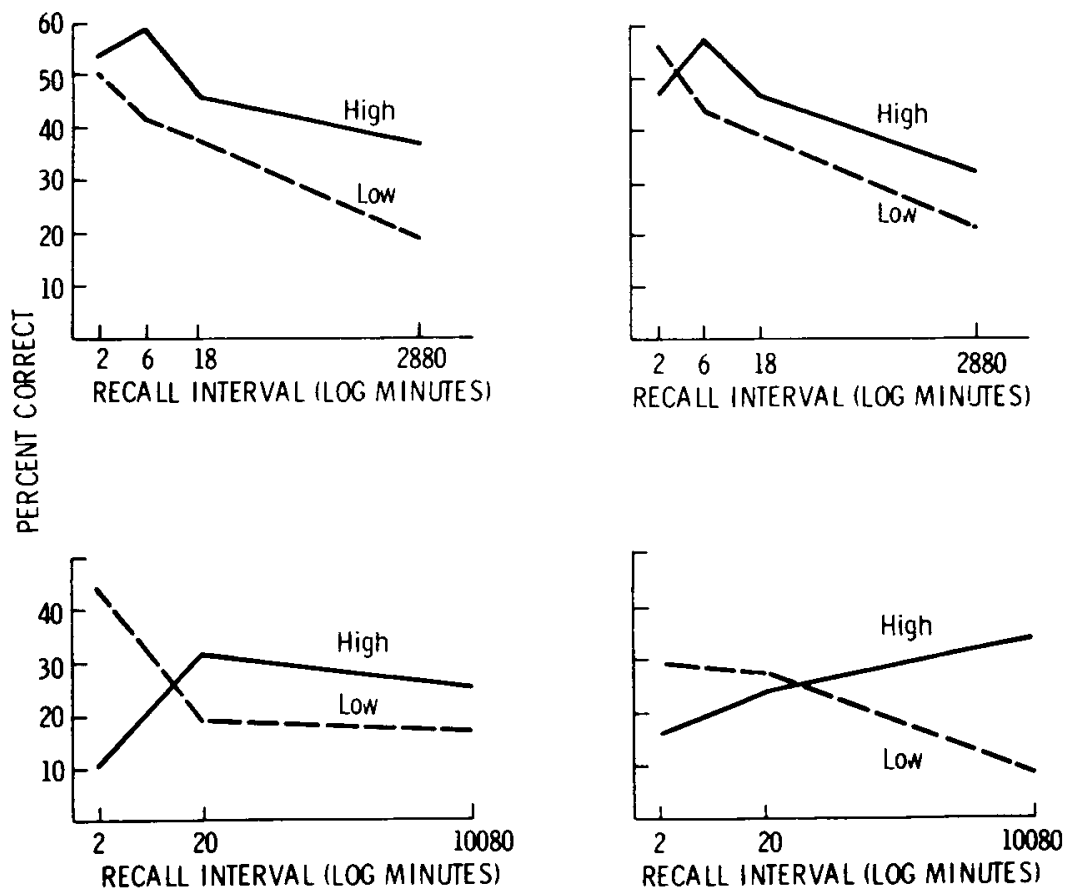

what might be called the "normative encoding" hypothesis.

The results presented here provide further evidence of the stability of both the within-S and the normative GSR effect in a one-trial paired-associate paradigm. These results also show that, for meaningful material, both normative and ipsative effects can be obtained in the same study.

\section{REFERENCES}

KAPLAN, R., \& KAPLAN, S. The arousal-retention interval interaction revisited: The effects of some procedural changes. Psychonomic Science, 1969, 15, 84-85.

KAPLAN, S., \& FISHER, G. R. A modified design for the Lykken zinc electrodes. Psychophysiology, 1964, 1, 88-89.

KAPLAN, S., \& HOBART, J. L. A versatile device for the measurement of skin resistance in rats and humans. American Journal of Psychology, 1964, 77, 309-310.

KAPLAN, S., KAPLAN, R., \& SAMPSON, J. R. Encoding and arousal factors in free recall of verbal and nonverbal material. Psychonomic Science, 1968, 12.73-74.

KLEINSMITH, L. J., \& KAPLAN, S. Paired-associate learning as a function of arousal and interpolated interval. Journal of Experimental Psychology, 1963, 65, 190-193. KLEINSMITH, L. J. \& KAPLAN, S. Interaction of arousal and recall in nonsense syllable paired-associate learning. Journal of Experimental Psychology, 1964, 67, 124-126. LEVONIAN, E. Short-term retention in relation to arousal. Psychophysiology, 1968, 4, 284-293.

MALTZMAN, I., KANTOR, W., \& LANGDON, B. Immediate and delayed retention, arousal, and the orienting and defense reflexes. Psychonomic Science, 1966, 6. 445-446.

POMERANTZ, J. R., KAPLAN, S., \& KAPLAN. $R$. Satiation effects in the perception of single letters. Perception \& Psychophysics, 1969.6, 129-132.

YARMEY, A. D. Word arousal and verbal mediation. Psychonomic Science, 1966, 6, 451-452.

\section{NOTES}

1. This investigation was supported by Research Grant MH-11599 from the National Institutes of Mental Health, United States Public Health Service. We are very grateful to Edward Levonian of the University of California, Los Angeles, for suggesting that we analyze our original data in terms of normative arousal categories.

2. The results of the 1963 and 1964 studies included for comparison here are somewhat different from the originally published ones. I or 
the 1963 study. the new plot is based on an ipative median split, as opposed to the three highest and three lowest items. lor both studies. Si with all-cero GSR, or only one xorable GSR have been eliminated. This prevents the problem of including a cro GSR in the "high-arousal" category. I or hoth studics, the revised plots are slightly less dramatic than the original ones, but the previously obtained significant interactions still hold.)

\section{The effect of type of redundancy on retention in preschool children ${ }^{1}$}

\begin{abstract}
DAVID A. CORSINI, University Georgia, Athens, Ga. 30601

The effect of verbal and nonverbal redundancy on the retention of 30 4-year-old children was examined to determine if the type of redundancy, as opposed to redundancy per se, facilitated the retention of this age child. The results demonstrated that the representational abilities of the child determine what type of redundant information will aid retention.
\end{abstract}

Three previous studies (Corsini, $\left.1969 a, b^{2}\right)$ found preschool children's retention of verbally presented information inferior to retention of information presented both verbally and nonverbally. The retention of older (second-grade) children was generally not different for these two ways of presenting information. These results have been discussed in terms of developmental changes in cognitive abilities for representation of information.

According to both Piaget (1947, and cv. Flavell, 1963) and Bruner (1964, 1966), the means by which the child can represent his environment changes with age. Developmentally, the child is first able to represent his environment only on the basis of his own physical movements; subsequently, the child becomes able to represent his environment through the use of imagery: and finally, the child becomes able to represent $h$ is experience symbolically. The primary hypothesis of the previous studies was that retention should be a joint function of the child's ability to represent information and the nature of the information presentcd. Thus, it was predicted that the preschool child, whose dominant means of representation is imagistic, would show better retention when the presented information allowed the use of imagery than when it did not; and additionally, this would not be the case for second-grade children who were capable of symbolic representation.

While the results of the previous studies have generally supported these predictions, the point can be raised that the preschool child's retention of information presented both verbally and nonverbally is superior not because the nonverbal information allows the preschool child to use imagery, but because a verbal-nonverbal message is simply more redundant than the verbal message. The present study asks whether the superior retention of preschool children for a verbal-nonverbal message is a function of simple redundancy (getting the message twice) or the specific type of redundancy (the nonverbal elements allowing the child to use imagery).

\section{GENERAL}

As in previous studies, $S$ was presented a simple instruction concerning the manipulation of familiar objects. The independent variable was the manner in which the instruction was presented. Retention was assessed nonverbally by requesting $S$ to perform the instruction with a set of objects made available after the instruction had been given.

\section{CONDITIONS}

\section{Verbal}

The $\mathrm{S}$ was given instructions only verbally. This assessed his ability to retain an entirely symbolic message.

$$
\text { Verbal-Nonverbal }
$$

The child was given instructions both verbally and nonverbally. If the instruction was, "Put the red car into the blue cup," the E held up a red car as he said "red car" and a blue cup as he said "blue cup." The objects were held up simultaneously with the verbal utterance but did not remain in S's view, i.e., no two objects were simultaneously in view.

\section{Verbal-Twice}

The $S$ was given instructions only verbally, as in the verbal condition, but was given the instruction twice before being asked to perform the instruction. This condition provided the child with redundant information-the instruction given twice-but the redundancy was only verbal.

The main comparison of interest is between the verbal-nonverbal condition and the verbal-twice condition. If the superior performance of preschool children in the verbal-nonverbal conditions of previous studies was a function of simple redundancy of information, then there should be no difference between these two conditions. However, if the young child retains verbally presented information poorly because he is cognitively less able to deal with symbolic information, then he should not profit significantly from receiving the verbal message twice.

\section{SUBJECTS}

Thirty preschool children (mean age $=$ 53.8 months) were obtained from the University of Georgia Laboratory Nursery School and a church-sponsored nursery school near the University of Georgia. There were 15 males and 15 females in the sample. The sample was predominantly white and from middle-class socioeconomic background.

STIMULI
The stimuli about which the to-be-retained instructions were given consisted of toy cars, crayons, cups, and boxes, each represented by three colors-red, yellow, and blue.

INSTRUCTIONS

Three levels of instructions were used in each condition. The levels differed in terms of the amount to be retained: two objects, three objects, and four objects. For example: Put the blue crayon in the red cup; put the blue car and yellow crayon in the red box; and put the yellow car in the blue cup and the red crayon in the yellow box.

\section{DESIGN}

Each $S$ received each of the conditions. The order of conditions was arranged so that $10 \mathrm{Ss}$ received each of the conditions first; 10 Ss received each of the conditions second, and $10 \mathrm{Ss}$ received each of the conditions third. This arrangement allowed for a between-Ss as well as a within-Ss analysis.

\section{SCORING}

Each of the objects used by $S$ was given a score of 1 if they had been mentioned in the instruction. If the pairing of objects was correct, an additional point was given in order to distinguish between partially correct and completely correct performance. For the three instructions given in each condition, the total possible score was 13 .

\section{PROCEDURE}

Each $S$ was asked to identify the objects and the colors used in the instructions. If $S$ was not sure of these, he was replaced. Four Ss were eliminated for this reason. The $S$ was then told he would be asked to do different things with the objects and was given one practice trial with the 